\title{
Transtorno do espectro autista e habilidades envolvidas no brincar: concepção de uma equipe multidisciplinar
}

\author{
Autistic spectrum disorder and skills involved in play: conception of a multiciplinary \\ Transtorno del espectro autista y habilidades implicadas en el juego: concepción de un \\ equipo multidiciplinario
}

Mayanny da Silva Lima ${ }^{1}$, Valeria Silva Carvalho ${ }^{1 *}$, Najra Danny Pereira Lima ${ }^{2}$, Marcus Vinicius da Rocha Santos da Silva ${ }^{1}$, Gilma Sannyelle Silva Rocha ${ }^{1}$, Thalia Costa Medeiros ${ }^{1}$, Thais Costa Medeiros $^{1}$, Maria Camila da Silva ${ }^{1}$, Mychelle Maria Santos de Oliveira ${ }^{1}$.

\begin{abstract}
RESUMO
Objetivo: Identificar quais os métodos ou procedimentos utilizados pelos profissionais nos atendimentos para desenvolver e potencializar as habilidades da criança autista através do brincar funcional. Métodos: Trata-se de um estudo avaliativo, exploratório, do tipo pesquisa ação, com abordagem qualiquantitativo. Participaram da pesquisa profissionais de uma equipe multiprofissional que atuam nos atendimentos no Centro de Atenção Psicossocial Infanto Juvenil (CAPS-IJ) com pacientes com Transtorno do Espectro Autista (TEA). A coleta de dados foi realizada com o uso de questionários com perguntas abertas e fechadas sobre o tema. Resultados: Diante das respostas obtidas, verifica-se o conhecimento superficial dos profissionais acerca da importância do brincar funcional no atendimento de crianças com TEA, pois ele auxilia no desenvolvimento total, configurando-se como fundamental no processo terapêutico, validando e potencializando as habilidades da criança, usufruindo do lúdico como ferramenta promotora de desenvolvimento. Conclusão: Os resultados demonstram o desconhecimento e a falta de capacitação para o ensino de habilidades por meio do brincar. Por meio deste estudo, enfatiza-se a importância do brincar no processo terapêutico e o conhecimento destes métodos pelos profissionais como ferramenta para aquisição de habilidades.
\end{abstract}

Palavras-chave: TEA, Brincar funcional, Equipe multidisciplinar.

\begin{abstract}
Objective: Identify which methods or procedures professionals use in care to develop and enhance the skills of the child diagnosed with autism spectrum disorder through functional play. Methods: This is an evaluative, exploratory, action research type study with a qualitative and quantitative approach. The place of the investigation was in a Child and Youth Psychosocial Service Center (CAPS-IJ). Professionals from a multiprofessional team who work in the CAPS-IJ services with patients with Autistic Spectrm Disorder (TEA) participated in the research. Data collection was performed using questionnaires with open and closed questions on the topic. Results: Given the responses obtained, the professionals' superficial knowledge about the importance of functional play in the care of children with ASD is verified, as it helps in the total development, becoming essential in the therapeutic process, validating and enhancing the skills. of the child, taking advantage of playfulness as a tool that promotes development. Conclusion: The results demonstrate the lack of knowledge and the lack of training for teaching skills through playing. through this study, the importance of playing in the therapeutic process and the knowledge of these methods by professionals as a tool for the acquisition of skills are emphasized.
\end{abstract}

Keywords: TEA, Functional play, Multidisciplinary team.

${ }^{1}$ Centro Universitário de Ciências e Tecnologia do Maranhão (UNIFACEMA), Caxias - MA.

*E-mail: valeriapsico28@gmail.com

2 Instituto Paradigma, São Paulo - SP. 


\section{RESUMEN}

Objetivo: Identificar los métodos o procedimientos utilizados por los profesionales en el cuidado para desarrollar y mejorar las habilidades del niño autista a través del juego funcional. Métodos: Se trata de un estudio de tipo investigación-acción, exploratorio y evaluativo, con enfoque cualitativo. En la investigación participaron profesionales de un equipo multiprofesional que laboran en los servicios CAPS-IJ con pacientes con transtorno del espectro autista (TEA). La recolección de datos se realizó mediante cuestionarios con preguntas abiertas y cerradas sobre el tema. Resultados: A la vista de las respuestas obtenidas, se verifica el conocimiento superficial de los profesionales sobre la importancia del juego funcional en el cuidado de niños con TEA, ya que ayuda en el desarrollo total, siendo fundamental en el proceso terapéutico, validando y potenciando las habilidades. del niño, aprovechando la alegría como herramienta que favorece el desarrollo. Conclusión: Los resultados evidencian el desconocimiento y la falta de formación para enseñar habilidades a través del juego. A través de este estudio se enfatiza la importancia del juego en el proceso terapéutico y el conocimiento de estos métodos por parte de los profesionales como herramienta para adquirir habilidades. Palabras clave: Alimentos Funcionales, Dieta, Enfermedad Crónica.

Palabras clave: TEA, Juego funcional, Equipo multidisciplinario.

\section{INTRODUÇÃO}

O Transtorno do Espectro Autista (TEA) é caracterizado como um transtorno de neurodesenvolvimento, com déficits clinicamente significativos na linguagem, interação social, bem como padrões restritos de comportamento e seletividade de interesses e atividades. Suas causas são desconhecidas, mas pode estar ligada a fatores ambientais ou genéticos (APA, 2014).

Os primeiros sinais de TEA aparecem logo antes dos 3 anos, como uma das características o anormal desenvolvimento da fala e a falta de interação da criança com o meio. Os prejuízos que se vinculam a restrita comunicação oral, e a sociabilidade, afeta significativamente outras áreas como cognitiva, educacional, social e o brincar (GUERRA BT e VERDU AC, 2016).

O brincar se estabelece como um dos fatores necessários para o desenvolvimento de uma criança, a brincadeira se constitui como um fenômeno universal que contribui positivamente para a maturação do indivíduo, favorecendo o desenvolvimento em diversos aspectos como coordenação motora, cognição, atenção, criatividade, contato com o meio externo, habilidades sociais, dentre outros (FONSÊCA ME e DA SILVA ÂC, 2018). Além disso, Klinger EF e Souza AP (2015), enfatiza que o brincar, possibilita a criança adentrar no mundo ilusório, de imaginação e fazer uma ponte com o que é real.

Ao que concerne $o$ ato de brincar, para crianças típicas se constitui como uma ação natural, onde uma se relaciona com a outra de forma simples e espontânea, e essa interação dá lugar a imaginação, abrindo caminhos e dando significado para a brincadeira por mais simples que ela seja. Enquanto para a criança com TEA, o ato de brincar não acontece dessa forma, emerge de forma diversa, pois as mesmas não interagem com outras crianças, desconheces o imaginário, praticadas lúdicas, além de pobreza de simbolização (BAGAROLLO M, et al., 2013).

O conhecimento do lúdico é de grande importância para desenvolvimento mental da criança com TEA, pois facilita a aprendizagem, além de apresentar o contexto social e cultural no qual ela vive, auxilia no processo de expressão, comunicação, socialização e integração, garantindo através do ato de brincar, a construção de vivencias, e potencialização de suas habilidades, aprendizagem de novos conceitos que lhe proporciona autonomia (ALBUQUERQUE OA, 2018).

O brincar auxilia diretamente no processo terapêutico da criança com TEA, comtemplando vários fatores do espectro, buscando atender através da ludicidade cada criança em suas particularidades. o brincar perde seu papel apenas de proporcionar lazer, pra assumir o posto de transformação na intervenção, contribuindo para o processo de neurodesenvolvimento, pois através do brincar a criança aprende, relaciona-se e comunica-se com o mundo (CIPRIANO MS e DE ALMEIDA MT, 2016). 
Mediante ao exposto, o interesse pelo tema surgiu diante de ser observado e estudado na literatura os atributos positivos que o brincar carrega consigo, e as contribuições para a aprendizagem e potencialização de habilidades. Nesta perspectiva, o presente estudo objetiva-se a identificar quais os métodos ou procedimentos os profissionais utilizam nos atendimentos para desenvolver e potencializar as habilidades da criança diagnosticada com TEA através do brincar funcional.

\section{MÉTODOS}

Trata-se de um estudo avaliativo, exploratório, do tipo pesquisa ação, com abordagem qualiquantitativa. A pesquisa-ação, conforme Tripp D (2005), é uma forma de investigação-ação que utiliza técnicas de pesquisa consagradas para informar a ação que se decide tomar para melhorar a prática. A pesquisa avaliativa é um procedimento que consiste em fazer um julgamento de uma intervenção usando métodos científicos.

O local da investigação foi em um Centro de Atendimento Psicossocial Infanto-Juvenil- CAPS-IJ, localizado no município do interior do Maranhão. Enfatiza-se que presente campo de pesquisa foi escolhido por oferecer atendimento para o público de interesse do estudo.

Participaram das pesquisas profissionais de uma equipe multiprofissional que atuam ativamente nos atendimentos no CAPS-IJ com crianças e adolescentes com TEA, bem como outras condições. Todos os profissionais atuantes no CAPS-IJ foram convidados a se fazer presente na pesquisa, mas somente 07 participaram, em um total de 14 profissionais. Para garantir o sigilo e anonimato, os nomes dos profissionais, foram substituídos pela primeira letra relacionada a seu cargo e numeração, caso fossem entrevistados mais profissionais de uma mesma categoria, exemplo: (Terapeuta Ocupacional - TO, Enfermeiros - E1, E2, Pedagogo- PG, Psicólogo - P, Assistente social - AS, Educador Físico- EF).

Os critérios de inclusão da pesquisa foram: ser profissional atuante no CAPS-IJ, ter o grau de escolaridade de nível superior ou técnico, atender criança com TEA e aceitar participar da pesquisa. Foram excluídos da pesquisa aqueles que não estavam em consonância com pelo menos um dos itens acima.

Quanto a coleta de dados, foi realizada mediante o uso de questionários com perguntas abertas e fechadas sobre os dados sociodemográficos dos participantes, bem como conhecimentos e práticas sobre 0 brincar funcional nos atendimentos com crianças diagnosticadas com TEA.

Ao que concerne risco e/ou desconfortos, a pesquisa não trouxe nenhum risco notável. Ao que se refere os benefícios propõe-se que com os resultados recolhidos dos profissionais do CAPS-IS, os mesmos adquiram conhecimento sobre o brincar, bem como sua importância e funcionalidade nos quadros de TEA, para reestruturar as atividades como meio de reforçar as habilidades dos pacientes atendidos no CAPS-IJ.

A pesquisa foi submetida a plataforma brasil e logo em seguida para o comitê de ética em pesquisa, onde obteve aprovação com o número de CAAE:97545018.5.0000.5554 e sob o Parecer: 3.101.304.

\section{RESULTADOS E DISCUSSÃO}

\section{Distribuição e descrição do perfil profissional dos sujeitos}

A Tabela 1 refere-se aos dados profissionais coletados a partir das entrevistas aplicadas aos profissionais atuantes no "Centro de Atenção Psicossocial Infanto Juvenil", localizado em uma cidade no interior do Maranhão, trata-se dos seguintes aspectos: Sexo, idade, profissão, especializações, tempo de serviço prestado na unidade e tempo de serviço com crianças com TEA. Assim, os achados dispostos nas tabelas foram separados em colunas, que demonstram as interlocuções de cada categoria profissional médicos, enfermeiros, psicólogo, terapeuta ocupacional, pedagogo e assistente social seguindo sempre a mesma ordem de apresentação: médicos $(M)$, enfermeiros $(E)$, psicólogo $(P)$, terapeuta ocupacional (TO), pedagogo (PG), assistente social (AS), conforme apresentados a seguir:

A seguir tem-se as características gerais dos participantes descrevendo os dados pessoais (sexo e idade); os dados profissionais (profissão, tempo de serviços na unidade e tempo de serviços com crianças com TEA): 
Tabela 1 - Características gerais dos participantes da pesquisa.

\begin{tabular}{|c|c|c|}
\hline Variáveis & Números & $\%$ \\
\hline \multicolumn{3}{|l|}{ Dados pessoais } \\
\hline \multicolumn{3}{|l|}{ Sexo } \\
\hline Masculino & 2 & $28,60 \%$ \\
\hline Feminino & 5 & $71,40 \%$ \\
\hline Total & 7 & $100 \%$ \\
\hline \multicolumn{3}{|l|}{ Idade } \\
\hline $24-29$ & 1 & $14,29 \%$ \\
\hline $30-35$ & 3 & $42,85 \%$ \\
\hline $36-41$ & 1 & $14,29 \%$ \\
\hline $42-47$ & 0 & 0 \\
\hline $48-53$ & 1 & $14,29 \%$ \\
\hline$>54$ & 1 & $14,29 \%$ \\
\hline Total & 7 & $100 \%$ \\
\hline \multicolumn{3}{|l|}{ Profissão } \\
\hline Médico & 0 & $0,00 \%$ \\
\hline Enfermeiro & 2 & $28,50 \%$ \\
\hline Psicólogo & 1 & $14,28 \%$ \\
\hline Terapeuta Ocupacional & 1 & $14,28 \%$ \\
\hline Pedagoga & 1 & $14,28 \%$ \\
\hline Assistente Social & 1 & $14,28 \%$ \\
\hline Educador físico & 1 & $14,28 \%$ \\
\hline \multicolumn{3}{|c|}{ Tempo de serviço na unidade } \\
\hline $1-<1$ anos & 2 & $28,57 \%$ \\
\hline 2- $1-4$ anos & 4 & $57,15 \%$ \\
\hline 3- 5-9 anos & 1 & $14,29 \%$ \\
\hline 4- >10anos & 0 & 0 \\
\hline \multicolumn{3}{|c|}{ Tempo de serviço com crianças com TEA } \\
\hline $1-<1$ ano & 5 & $71,42 \%$ \\
\hline 2- $1-4$ anos & 1 & $14,29 \%$ \\
\hline 3- 5-9 anos & 1 & $14,29 \%$ \\
\hline$>10$ anos & 0 & 0 \\
\hline
\end{tabular}

Fonte: Lima MS, et al., 2021.

A partir da análise dos dados identificou-se que $71,4 \%$ da amostra foi composta por participantes do sexo feminino, o sexo masculino representou $28,6 \%$ da amostra do estudo. A média de idade dos participantes foi de 30 a 35 anos de idade. Em relação aos dados profissionais é possível notar que $28,5 \%$ da amostra do estudo foi composta por enfermeiros e as demais profissões representaram 14,28\% dos participantes. Em pauta disso, para Bossato HR, et al. (2020), a presença do profissional de enfermagem na equipe do Caps é extremamente relevante pois se articulam com outros serviços, com as famílias e dispositivos comunitários, fortalecendo a rede de apoio do usuário do serviço de saúde mental.

No que diz respeito ao tempo de serviço prestado na instituição os dados mostram que os profissionais participam da equipe a cerca de 1 a 4 anos. No que concerne atendimento a crianças com TEA, apenas 1 participante relatou já possuir mais tempo de atendimento e experiência com esse público. Reitera-se que o atendimento a pessoas com TEA requer uma intervenção direcionada e intensiva (LOVAAS OI, 1987). E por isso faz-se necessário a presença de uma equipe multiprofissional preparada e experiente nesses atendimentos pois a falta de experiência da equipe pode impactar negativamente no desenvolvimento da criança (PORTOLESE J, et al., 2017). 
Nesse sentido, para Furtado L, et al. (2014), tanto pessoas que possuem diagnóstico de TEA quanto sua família, têm direito ao acolhimento e cuidado integral no Sistema Único de Saúde (SUS), do qual faz parte os CAPS. E desse modo, segue a importância da equipe se manter preparada e qualificada nesses atendimentos pois o SUS é porta de entrada e o meio de acesso mais procurado por pais de pessoas com o diagnóstico de TEA.

Em relação aos profissionais que compõem a equipe, os dados mostram que é formada por: enfermeiros, pedagoga, psicóloga, assistente social, educador físico e terapeuta ocupacional. No que diz respeito a isso, as famílias relatam que essas necessidades de serviço muitas vezes não são atendidas

\section{Conhecimento dos profissionais a respeito do Brincar Funcional e sua importância para o desenvolvimento da criança}

Nessa categoria, foi questionado aos participantes o que eles entendem por brincar funcional, logo, os profissionais revelaram as seguintes percepções ao que se refere a temática:

"É uma forma adaptada de brincar p/ criança aprender e socializar" (AS).

"O brincar que auxilia a criança a desenvolver habilidades afetivas $e$ cognitivas" (E1).

"Brincar que direciona a algo que leve ao aprendizado e desenvolvimento da criança" (P).

"Brincar de forma adequada" (PG).

"Que a criança apresente funcionalidade ao brincar ex: com um carrinho empurrando e fazendo VRUM, UM" (TO).

"Faço trabalho individual ou em grupo trabalhando a lateralidade, coordenação motora e a socialização entre eles através do brincar" (EF).

"O brincar funcional é aquele que ajuda a criança a desenvolver habilidades que não tinha e/ou potencializar as habilidades positivas para o desenvolvimento motor, pessoal e social" (E2).

Em face do exposto, averígua-se que os interlocutores parecem demonstrar pouca compreensão acerca do contexto interrogado. O brincar é uma habilidade crucial no desenvolvimento de uma criança, incluindo aqueles com necessidades especiais. Através do brincar, as crianças são apresentadas aos conceitos essenciais na linguagem, alfabetização e outras oportunidades importantes para o desenvolvimento (BARBOSA MO e FUMES NLF, 2017).

O entrelaçamento entre o imaginar e realizar leva a criança a um estado de livre desenvolvimento, aprimorando suas competências, desafiando suas capacidades e adquirindo experiências frente ao meio no qual convive. A partir disso, obtém possíveis oportunidades de se expressar e ser compreendido. É no ato de brincar que a criança desempenha oportunidades de constituir o seu EU, permitindo a formação e expressão dos seus desejos e vontades (COELHO R e TADEU B, 2015).

Para Azevedo MFN (2015), o brincar favorece uma melhora no desenvolvimento de crianças que apresentam o diagnóstico de TEA. Além disso, é uma ferramenta que possibilitam elas a identificar e aprender atividades do dia-dia e a conhecerem o seu próximo, realizando troca de afetividade e desenvolvimento de papéis e regras. Essa possibilidade torna-se valiosa, uma vez que criança com autismo possui particularidades que direcionam a prejuízos nos aspectos supracitados. Enfatiza-se que a criança com esse diagnostico deve receber instruções sucintas, suporte visual para compreensão da brincadeira, além disso o brincar deve considerar os interesses individuais da criança.

Além disso, foi indagado aos participantes se eles consideram o brincar funcional importante para o desenvolvimento da criança com TEA. No que se referem a tal questionamento os interlocutores revelaram as seguintes concepções: 
"Sim, porque através do brincar a criança aprende e coloca em pratica as atividades da vida diária (AVDs) comer, vestir, escovar os dentes" (TO).

"Sim... para desenvolver a alta estima e a socialização através do brincar" (EF).

"Sim, por que é uma forma de estimulação" (PG).

"Sim, contribui na aprendizagem e desenvolvimento da criança" (P).

"Sim, desenvolvimento da comunicação, melhora na afetividade e comportamento". (E1).

"Sim, no momento da adaptação ele vai se desenvolver melhor, vai ter mais socialização" (AS).

"Sim. Uma pessoa que tem atraso no desenvolvimento, quanto mais recursos, melhor. Aprender brincando é um barato com essas brincadeiras, a criança pode aprender as habilidades escolares, motoras, comunicacionais e sociais" (E2).

Observa-se que os profissionais exibem algum conhecimento sobre a importância da brincadeira como forma de aprendizagem e desenvolvimento de habilidades. De acordo com a literatura, o ato de brincar norteia a criança para uma socialização permitindo um contato direto com o meio externo de forma lúdica e recreativa, possibilitando através da simbolização adquiridos ao brincar uma aproximação maior com a realidade (PEREIRA C, et al., 2013; CHIOTE FA, 2013). Desse modo, o brincar pode apresentar-se de forma atípica, isto é, a forma como a criança manipula os brinquedos e os movimentos que ela realiza durante o brincar é compreendido como estereotipados.

No que diz respeito a importância da utilização de recursos metodológico para influenciar no desenvolvimento, foi questionado aos profissionais sobre a utilização de instrumentos para identificar habilidades envolvidas no brincar com crianças com TEA, ao que concerne a isso, os profissionais emitiram as seguintes afirmações:

"Sim, utilizo uma caixa lúdica com bonecos p/ ver o interesse para a criança começar" (AS).

"Não" (E2).

"Não... Apenas utilizo o brincar através do tempo de convivência com o paciente[...]" (EF).

Ao analisar as falas dos interlocutores, observar-se que os mesmos demonstram conhecimento superficial ao que se refere a temática. Sobre isso, reitera-se que 0 atendimento a crianças com TEA precisa ser estruturado (isto é, organizado visualmente indicando à criança o início, meio e fim da atividade) (DEFENSENETRVAL DA e FERNANDES FD, 2016).

O conhecimento dos profissionais sobre pré-requisitos e recursos metodológicos faz toda diferença no prognóstico do quadro, pois a partir isso, são criadas e traçadas novos métodos e estratégias de ensino viabilizando o desenvolvimento da criança, e potencializando suas habilidades. Dessa forma, quanto maior $o$ nível de conhecimento da equipe multiprofissional sobre o processo educativo e recursos disponibilizados, maiores são as probabilidades de aprendizagens (BARBOSA MO e FUMES NLF, 2017).

Foi alvo de interesse deste estudo, além do que foi descrito até aqui, investigar os conhecimentos dos profissionais sobre os requisitos básicos para brincar (e que em geral as crianças com TEA possuem dificuldade em desenvolver), bem como os procedimentos usados pelos profissionais, para avaliar e treinar estas habilidades. Sobre isso, os dados encontrados serão apresentados a seguir:

"Não diferencio nenhuma criança. Pois uma criança com TEA pode fazer qualquer coisa como uma criança normal [...]" (EF). 
"Deixar a criança a vontade no espaço, transmitir confiança e auxiliá-la no sentido de deixar todos os recursos a sua disposição para assim usá-los como for do seu desejo" (P).

"Quando não identifico nenhum pré-requisito encaminho $p$ outros profissionais mais indicados" (AS).

Sobre essa questão é notório que os profissionais exibem respostas superficiais sobre o assunto, demostrando desconhecimento sobre o tema em questão. Segundo Lovaas OI (1987), o passo inicial e fundamental para o ensino de habilidades em pessoas com atrasos de desenvolvimento é ensinar prérequisitos necessários e básicos para o desenvolvimento de tarefas simples, por exemplo "sentar", "levantar" e "manter contato visual", para que posteriormente repertórios mais complexos possam vir fazer parte da vida do indivíduo.

Com isso, se faz de suma importância que o profissional tenha conhecimento sobre procedimentos usados no brincar para identificar e potencializar as habilidades já existente, bem como ensinar as que ainda não fazem parte do repertório da criança (SANINI C, et al., 2013). Pois o profissional constitui-se como um mediador no processo de aprendizagem, guiando a criança pelo melhor caminho para assim obter êxito no processo terapêutico (DINIZ FJ, 2017).

Os resultados têm mostrado que quando o ensino de repertório é bem estruturado, vários operantes como comportamentais, verbais e cognitivos podem ser ensinados para essa população, a partir da aprendizagem de repertórios com um certo nível de complexidade, a pessoa com TEA torna-se mais autônomo (GUERRA BT e VERDU AC, 2016).

Para mais, indagou-se os profissionais sobre a utilização de critérios que levariam a obter as melhores escolhas de utilização de brinquedos e brincadeiras a ser desenvolvida com o paciente com TEA, em detrimento a isso, eles revelaram que:

"Apenas encaminho $\mathrm{p}$ / outros profissionais que possam desenvolver atividades com eles, quando percebo que ele precisa de um acompanhamento mais especifico"(AS).

"Como falei antes faço trabalho em grupo e individual. Faço o trabalho individual com todo o tipo de grau de TEA mais sempre tentando fazer com que eles se socializem junto aos outros, independente de qual grau de TEA ele possa ter[...]"(EF).

"A primeira coisa a ser feita é conhecer a criança, observar as principais dificuldades, traçar objetivos, e planejar as atividades que vão servir para desenvolver aquelas habilidades [...]. Só depois se pode escolher brinquedos e brincadeiras que são funcionais" (E1).

Mediante o exposto, nota-se que os profissionais participantes apresentam pouco ou mesmo não têm conhecimento acerca da importância de selecionar brinquedos e brincadeiras para assim trabalhar com a criança com TEA. É possível verificar ainda, que grande parte dos profissionais preferiram não emitir suas respostas acerca do que foi questionado, tendo em vista não compreender a forma de seleção de brinquedos e brincadeiras e os mesmos não realizam em sua prática diária. Segundo Fernandes A, et al. (2018), é por base de brincadeiras, fantasias e faz - de - conta que a pessoa com TEA atribui significados a pessoas, objetos e lugares. Pois o brincar por sua natureza é um meio socializador.

Corroborando ao exporto, Campos C, et al. (2018), aponta que o brincar é uma técnica usada por profissionais de diferentes áreas de atuação que torna o atendimento mais eficaz se tratando de crianças, e principalmente aquelas que apresentam dificuldades de aprendizagem. Além disso, para maximizar algumas habilidades da criança com TEA como a imitação, as funções de alguns objetos e a simbolização, a brincadeira seria uma técnica bastante eficaz pois traria melhoras na aprendizagem, inclusive no ambiente escolar. 
Segundo Albuquerque OA (2018), a brincadeira tem um papel primordial na inclusão da pessoa com TEA, proporcionando um bom relacionamento de uma criança com a outra, através do compartilhamento de brinquedos e brincadeiras, despertando a imaginação e emergindo uma colaboração entre os pares. A forma de trabalhar de forma lúdica, torna o processo terapêutico mais cativante, fazendo com que a criança cm TEA se interesse pela atividade proposta, e aumenta positivamente as chances do seu desenvolvimento.

Faz parte do processo terapêutico que o profissional selecione brinquedos e brincadeiras para que a criança se sinta estimulada pelo que é lhe apresentado, onde ele irá ajudar na aquisição de competências, ressaltando que essa escolha se dá através da observação do desenvolvimento das potencialidades, para que a mesma possa acompanhar o que é proposto através do lúdico no atendimento. Para mais, autores afirmam que a ludicidade é de extrema importância para o desdobramento da mente infantil, ela facilita a aprendizagem o desenvolvimento cultural, contribuindo significativamente com vários processos importantes na vida da criança como: social, pessoal e comunicacional (MORAIS TL, 2012; KORTMANN GM, 2013).

\section{CONCLUSÃO}

Os resultados deste estudo mostram que os profissionais demonstram pouco conhecimento ao que se refere o brincar e suas potencialidades no atendimento de crianças com TEA, bem como a falta de capacitação para o ensino de habilidades a partir do brincar, já que o mesmo proporciona o desenvolvimento da criança, além de promover diversos tipos de aprendizagem como a linguagem, alfabetização, bem como as atividades de rotina, e a potencialização de habilidades já existentes visando a autonomia da criança como um todo, apresentando o meio externo e as relações sociais de forma lúdica. Conclui-se que é necessário que os profissionais busquem métodos de qualificação necessários sobre o ensino de habilidades para crianças com TEA através do brincar.

\section{REFERÊNCIAS}

1. ALBUQUERQUE OA. A utilização dos jogos e das brincadeiras na educação infantil para crianças com transtorno do espectro autista. 2018. Monografia (obtenção do grau de licenciado em Pedagogia)- Faculdade Federal da Paraíba, João Pessoa, 2018; 43p.

2. APA. American Psychiatric Association. Diagnostic and statistical manual of mental disorders. 2014. Disponível em: https://www.psychiatry.org/psychiatrists/practice/dsm. Acessado em: 24 de Janeiro de 2021.

3. AZEVEDO MFN. A importância de brincar para o desenvolvimento de crianças com perturbação do Espectro do Autismo. 2015. Dissertação. (Mestrado em ciência da educação na especialidade em educação especial: domínio cognitivo motor)- Escola de Superior de Educação João de Deus, Lisboa, 2015; 98p.

4. BARBOSA MO, FUMES NLF. Atividade docente e reflexões no atendimento educacional especializado para estudantes com transtorno do espectro autista. Revista Educação e Cultura Contemporânea, 2017; 14: e1.

5. BAGAROLLO M, et al. An Autistic Child's Play From The Cultural-historical Perspective [o Brincar De Uma Criança Autista Sob A ótica Da Perspectiva Histórico-cultural]. Revista Brasileira de Educação Especial, 2013; 19: $107-120$.

6. BOSSATO HR, et al. A enfermagem e o protagonismo do usuário no CAPS: um estudo na perspectiva construcionista. Revista Gaúcha de Enfermagem, 2020; 42.

7. CAMPOS $\mathrm{C}$, et al. Expectativa de profissionais da saúde e de psicopedagogos sobre aprendizagem e inclusão escolar de indivíduos com transtorno do espectro autista. Revista Psicopedagogia, 2018; 35: 3-13.

8. CHIOTE FA. A mediação pedagógica no desenvolvimento do brincar da criança com autismo na educação infantil. PRÓ-DISCENTE, 2013; 19.

9. CIPRIANO MS, DE ALMEIDA MT. O brincar como intervenção no transtorno do espectro do autismo. Extensão em Ação, 2016; 2.

10. COELHO R, TADEU B. A importância do brincar na educação de infância. Atas do Il encontro de mestrados em educação e ensino da Escola Superior de Educação de Lisboa, p. 106-114, 2015.

11. DEFENSE-NETRVAL DA, FERNANDES FD. A oferta da terapia fonoaudiológica em locais de assistência a indivíduos com Transtornos do Espectro do Autista (TEA). Sociedade Brasileira de Fonoaudiologia, 2016; 28.

12. DINIZ FJ. Autismo, ambiente escolar e obstáculos no processo de ensino-aprendizagem. Monografia (Trabalho de Conclusão de Curso) - Universidade Federal do Rio Grande do Norte, 2016; 20p.

13. FELISBERTO E, et al. Institucionalizando a avaliação nas organizações e agências de pesquisas: um estudo de caso exemplar. Saúde em Debate, 2017; 41: 387-399. 
14. FERNANDES A, et al. A criança com transtorno do espectro autista (TEA). Revista de Terapia Ocupacional da Universidade de São Paulo. 2018; 29: 187-194.

15. FONSÊCA ME, DA SILVA ÂC. Concepções e uso do brincar na prática clínica de terapeutas ocupacionais/Conceptions and use of play in occupational therapists clinical practice. Cadernos Brasileiros de Terapia Ocupacional, 2018; 23: 589-597.

16. FURTADO L, et al. Transtornos globais de desenvolvimento e saúde pública: uma revisão integrativa. Rev. Percurso Acadêmico, 2014; 4: 283-297.

17. GUERRA BT, VERDU AC. Ensino de operantes verbais em pessoas com Transtorno do Espectro Autista no The Analysis of Verbal Behavior: revisão sistemática. Revista Brasileira de Terapia Comportamental e Cognitiva, $2016 ; 18$.

18. KLINGER EF, SOUZA AP. Análise clínica do brincar de crianças do espectro autista. Distúrbios da Comunicação, $2015 ; 27$.

19. KORTMANN GM. Aprendizagem da criança autista e suas relações familiares e sociais: estratégias educativas. Monografia (Especialização em Neuropsicologia) - Instituto de Psicologia. Universidade Federal do Rio Grande do Sul, Porto Alegre, 2013; 33p

20. LOVAAS OI. Behavioral treatmentand and educational and intellectual functioning in young autistic children. Journal of Consulting and clinical psychology, 1987; 55(1).

21. MORAIS TL. Intervenção Pedagógica em Crianças com Perturbações do Espectro Autista. Escola Superior de Educação Almeida. Dissertação (Educação Especial) - Escola Superior de Educação, Lisboa, 2012; 10p.

22. OLIVEIRA S, et al. Mediação do brinquedo na comunicação com crianças com transtornos do espectro autista. 2019.

23. PEREIRA C, et al. Autismo infantil: aplicações do ensino estruturado na inclusão escolar. Revista de Ciências da Saúde Nova Esperança, 2013; 11: 88-95.

24. PORTOLESE J, et al. Mapeamento dos serviços que prestam atendimento a pessoas com transtorno do espectro autista no Brasil. Cadernos de Pós-Graduação em Distúrbios do Desenvolvimento, 2017; 17: 79-91.

25. SANINI C, et al. Competência social e autismo: o papel do contexto da brincadeira com pares. Psicologia: Teoria e Pesquisa, 2013; 29(1): 99-105, 2013.

26. TRIPP D. Pesquisa-ação: uma introdução metodológica. Educação e pesquisa, 2005; 31(3). 